\title{
Kopftrauma bei der Katze
}

\author{
Michaele Alef
}

Der Kopf ist bei der traumatisierten Katze nach den Gliedmaßen die am zweithäufigsten betroffene Region. Kieferfrakturen machen ca. $20 \%$ aller Frakturen bei der Katze aus. Ursachen sind Autounfälle, Fensterstürze und andere unbekannte stumpfe Traumata, aber auch Schussverletzungen [21]. Die entscheidende Frage bei diesen Patienten ist, ob es sich „nur“ um eine Verletzung der Weichteile und/oder der Knochen handelt oder ob auch das Gehirn betroffen ist. Die Differenzierung zwischen Kopfverletzung und SchädelHirn-Trauma (SHT) ist wichtig, da sowohl die Prognose als auch die notwendigen therapeutischen Überlegungen sehr unterschiedlich sind.

\section{Diagnose SHT - nicht immer einfach zu stellen}

Viele der möglichen Symptome einer Beteiligung des Zentralnervensystems ( $\triangleright$ Tab. 1) sind beim Tier nur schwer oder nicht nachvollziehbar. Die meisten übrigen Symptome - wie die Befunde am Auge, eine Bradykardie oder ein Lungenödem können auch andere Ursachen haben.

\section{Der intrakranielle Druck als entscheidender Parameter}

Die durch ein Trauma des Zentralnervensystems verursachte akute neuronale Schädigung kann therapeutisch nicht beeinflusst werden. Vielmehr zielen therapeutische Maßnahmen darauf, Sekundärschäden zu verhindern bzw. zu minimieren. Diese werden durch diverse komplexe pathophysiologische Prozesse verursacht, die zu einer nicht mechanischen neuronalen Schädigung führen. Diese kann innerhalb von Minuten bis Tagen nach dem Insult auftreten.

Ein für Entstehung und Ausmaß der Sekundärschäden entscheidender Parameter ist der intrakranielle Druck. Dessen zentrale Rolle ist durch die einmalige anatomische Situation im Schädel bedingt ( $\triangleright$ Abb. 1). Das durch Gewebe, Blut und Liquor eingenommene Volumen ist wegen der festen Schädelkapsel unveränderlich. Eine Raumforderung durch eine Blutung, ein Ödem oder auch eine Vasodilatation muss eine Volumenminderung an einer anderen Stelle/eines anderen Kompartiments zur Folge haben (Monro-Kellie-Doktrin). Möglich ist eine gewisse Volumenverschiebung der flüssigen Komponenten (nur ca. 16\% des Inhalts des Neurokraniums), so kann Liquor in begrenztem Ausmaß in Richtung Wirbelsäule verschoben oder die Perfusion des Hirngewebes vermindert werden (Kompensationsphase). Sind diese beschränkten Kompensationsmechanismen erschöpft, kommt es zu einem intrakraniellen Druckanstieg. Nach der Erschöpfung der Ausgleichsräume des intrakraniellen Kompartiments droht ein Circulus vitiosus ( Abb.2). Steigt der intrakranielle Druck, der dem arteriellen Blutdruck als zu überwindender Widerstand entgegensteht, sinken der zerebrale Perfusionsdruck und damit die Durchblutung des Gehirns. Der Körper versucht, der Minderperfusion des Gehirns entgegenzuwirken und senkt den Widerstand der

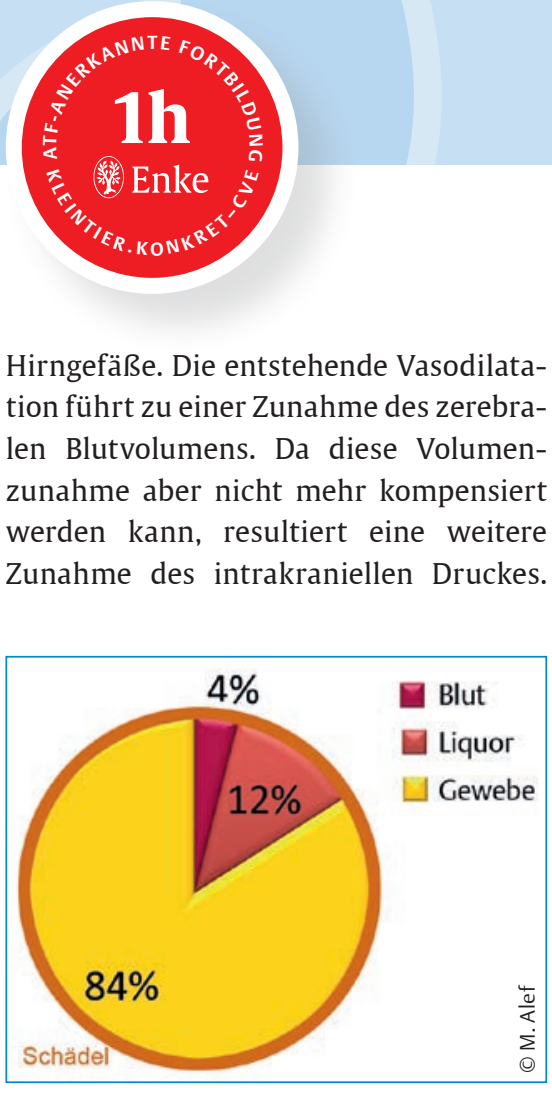

Abb. 1 Inhalt des Neurokraniums, MonroKellie-Doktrin.

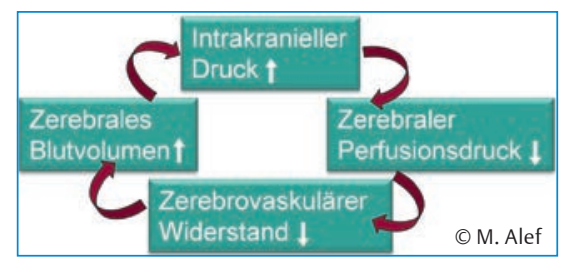

Abb. 2 Circulus vitiosus nach Erschöpfung der Ausgleichsräume des intrakraniellen Kompartiments.

Tab. 1 Mögliche Symptome eines Schädel-Hirn-Traumas (SHT).

\begin{tabular}{|c|c|}
\hline $\begin{array}{l}\text { Für die Diagnosestellung beim Tier } \\
\text { verfügbar }\end{array}$ & $\begin{array}{l}\text { Für die Diagnosestellung beim Tier } \\
\text { nur eingeschränkt verfügbar }\end{array}$ \\
\hline $\begin{aligned} & \text { - } \text { Bewusstseinsstörung, zunehmende Eintrübung, } \\
& \text { Bewusstlosigkeit } \\
& \text { - } \text { Vomitus } \\
& \text { - } \text { Gleichgewichtsstörungen } \\
& \text { - } \text { Anisokorie, Miosis, Mydriasis, Schielen } \\
& \text { - Krämpfe, Status epilepticus } \\
& \text { - Spastizität der Extensormuskulatur } \\
& \text { (Enthirnungsstarre) } \\
& \text { - Papillenödem } \\
& \text { - } \text { Lungenödem } \\
& \text { - Bradykardie, Arrhythmien ( } \rightarrow \text { Cushing-Reflex) }\end{aligned}$ & $\begin{array}{l}\text { - Kopfschmerz } \\
\text { - Nausea } \\
\text { - Schwindel } \\
\text { - Wortfindungsstörungen } \\
\text { - Amnesie } \\
\text { - visuelle Halluzinationen }\end{array}$ \\
\hline
\end{tabular}


- Abb. 3 verdeutlicht die dramatischen Folgen.

In der terminalen Phase folgt der intrakranielle Druck passiv dem arteriellen Blutdruck, der zerebrale Blutfluss sistiert und der Hirntod folgt.

\section{Therapieprinzipien}

Der Zusammenhang von zerebralem Perfusionsdruck (CPP), mittlerem arteriellen Blutdruck (MAP) und intrakraniellem Druck (ICP) ist wichtig für die Ableitung des basalen Therapieprinzips.

$\mathrm{CPP}=\mathrm{MAP}-\mathrm{ICP}$

Um die Perfusion des Gehirns zu gewährleisten, gilt es, den Blutdruck zu erhalten oder zu steigern und/oder den intrakraniellen Druck zu senken.

\section{Cushing-Reflex}

Die Beziehung zwischen Blutdruck, intrakraniellem Druck und zerebralem Perfusionsdruck erklärt auch den Cushing-Reflex, dessen Symptome Bradykardie (nicht obligat) und hoher Blutdruck bei einem Traumapatienten auf das Vorliegen eines SHT hinweisen können. Steigt der intrakranielle Druck, kommt es zu einer zerebralen Hypoxie. Um diesem Phänomen vorzubeugen, wird der systemische Blutdruck vom Organismus oft dramatisch gesteigert. So versucht dieser den zerebralen Perfusionsdruck und damit die Durchblutung des Gehirns aufrechtzuerhalten. Dadurch erhöht sich jedoch wiederum der intrakranielle
Druck (folgt in der Dekompensation dem Blutdruck) und der Blutdruck muss weiter steigen. Dramatische Blutdrucksteigerungen bis $300 \mathrm{mmHg}$ sind möglich. Reflektorisch kann die Herzfrequenz bis zu einer Bradykardie sinken.

Zusätzlich können Arrhythmien, eine unregelmäßige Atmung und ein Lungenödem (häufig in den kaudodorsalen Lungenbezirken) auftreten. Die Pathogenese dieser Symptome ist nicht vollständig geklärt, möglicherweise spielt eine Beeinträchtigung der Hirnstamm-Funktion eine entscheidende Rolle.

\section{.konkret}

Hat ein Patient mit offensichtlicher oder möglicher Kopfverletzung eine Bradykardie, kann ein SHT mit erhöhtem intrakraniellem Druck vorliegen!

\section{Zerebraler Blutfluss}

Die Durchblutung des Gehirns bleibt über einen weiten Blutdruckbereich von 50-150 mmHg (mittlerer arterieller Blutdruck, MAP) konstant ( Abb.4). Diese Autoregulation garantiert Funktion und Integrität des Hirngewebes unabhängig von akuten, „normalen“ Blutdruckschwankungen. Sinkt der arterielle Mitteldruck unter etwa $50 \mathrm{mmHg}$, fällt der zerebrale Blutfluss fast linear steil ab, bis die Durchblutung des Gehirns vollständig sistiert. Bei einem arteriellen Mitteldruck von über $150 \mathrm{mmHg}$ steigt der zerebrale Blutfluss hingegen steil an. Als
Folge nimmt der Anteil des Blutes am Inhalt des Neurokraniums zu (Anstieg des zerebralen Blutvolumens), eine Kompensation ist notwendig. Da nur der Liquor als Ausgleichskompartiment zur Verfügung steht, ist dieser Kompensationsmechanismus bald erschöpft und ein Anstieg des intrakraniellen Druckes ist die Folge.

Solange der arterielle Sauerstoffpartialdruck $\left(\mathrm{PaO}_{2}\right)$ höher als $50 \mathrm{mmHg}$ ist, beeinflusst er den zerebralen Blutfluss ebenfalls nicht. Droht bei niedrigeren Werten eine Sauerstoffminderversorgung des Gehirns, steigert der Körper den zerebralen Blutfluss drastisch. Auch hier drohen dieselben negativen Konsequenzen wie oben geschildert: Die $\mathrm{Zu}$ nahme des intrazerebralen Blutvolumens muss kompensiert werden, ist dies nicht möglich, steigt der intrakranielle Druck.

Zwischen dem arteriellen Kohlendioxidpartialdruck $\left(\mathrm{p}_{\mathrm{a}} \mathrm{CO}_{2}\right)$ und dem zerebralen Blutfluss hingegen besteht über einen weiten Bereich eine nahezu lineare positive Korrelation. Steigt der Kohlendioxidpartialdruck, steigt auch die Durchblutung des Gehirns. Erreicht wird dies über eine zerebrale Vasodilatation. Bei einem Abfall des arteriellen Kohlendioxidpartialdrucks wird die Durchblutung des Gehirns mithilfe einer zerebralen Vasokonstriktion gedrosselt. Dieser Mechanismus birgt große Gefahren für unsere Patienten ( $\triangleright$ s. Kasten 1 ), aber auch die Möglichkeit einer relativ einfachen Beeinflussung der Hirndurch-

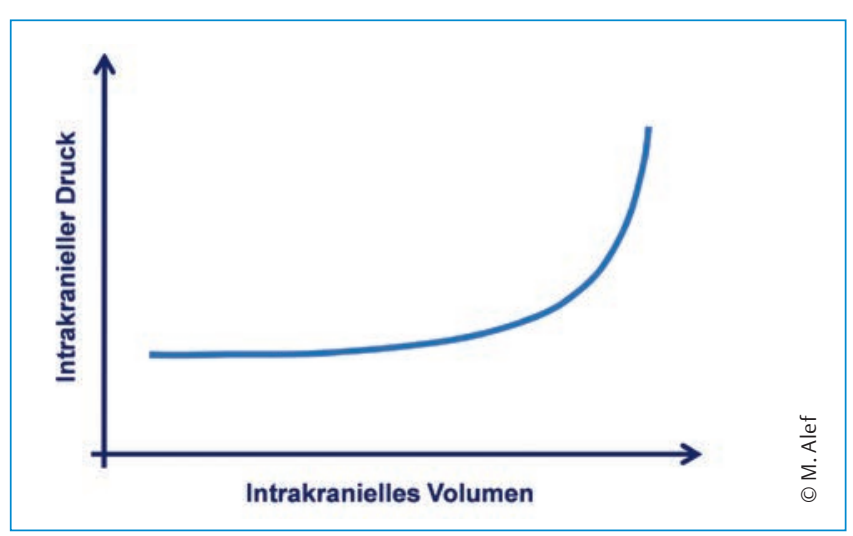

Abb. 3 Beziehung zwischen intrakraniellem Volumen und intrakraniellem Druck.

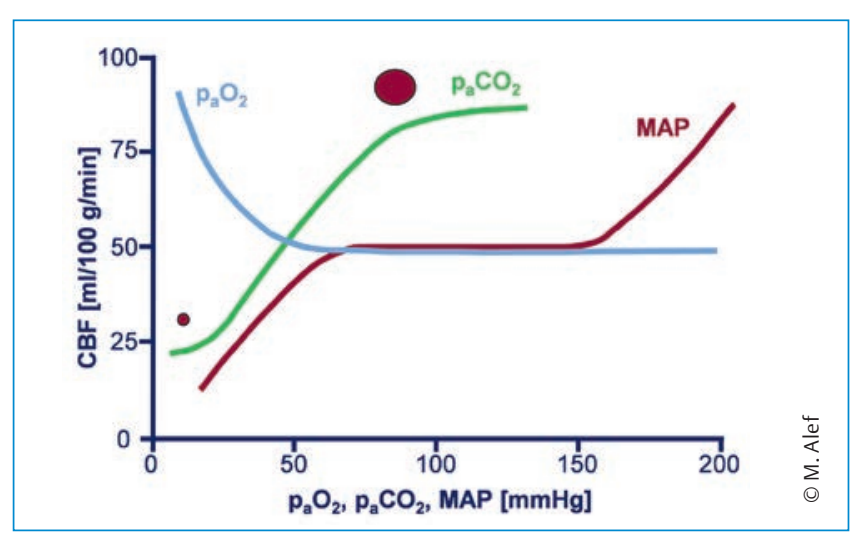

Abb. 4 Abhängigkeit des zerebralen Blutflusses (CBF) vom mittleren arteriellen Blutdruck (MAP), dem arteriellen Sauerstoff- $\left(\mathrm{p}_{\mathrm{a}} \mathrm{O}_{2}\right)$ und Kohlendioxidpartialdruck $\left(\mathrm{p}_{\mathrm{a}} \mathrm{CO}_{2}\right.$, jeweils in $\mathrm{mmHg}$ ). Schematisch dargestellt ist außerdem die Abhängigkeit des Durchmessers der zerebralen Blutgefäße (rote Punkte) vom arteriellen Kohlendioxidpartialdruck. 
blutung über den arteriellen Kohlendioxidpartialdruck, der wiederum durch Beatmung recht einfach variiert werden kann.

Bei einem SHT sollten die sogenannten 3 „malignen $\mathrm{H}^{\text {“ }}$ unbedingt vermieden werden:

- Hypoxie

- Hypotonie/Hypertonie

- Hyperkapnie

\section{Diagnostik und prognostische Faktoren}

Kommt zu den Weichteil- und/oder Knochenverletzungen am Kopf noch ein SHT dazu, verschlechtert sich die Prognose des Patienten merklich. Die Behandlung wird aufwändiger und damit auch kostenintensiver. Um Diagnostik und Therapie adäquat planen zu können, aber auch um ein fundiertes Besitzergespräch führen zu können, gilt es, nach der Einschätzung des Allgemeinzustands $(\rightarrow$ initiale Notfallmaßnahmen) die Schwere der neurologischen Schädigung zu beurteilen und nach weiteren für Prognose und Therapie relevanten Verletzungen zu suchen [13]. Solche Begleitverletzungen erhöhen u.U. nicht nur den therapeutischen Aufwand und damit die zu erwartenden Kosten immens, sie können auch für die Prognose des Patienten noch bedeutender sein als das SHT selbst. So kann ein stumpfes Thoraxtrauma akut lebensbedrohlich sein, eine abdominelle Verletzung eine baldige Operation notwendig machen und ein Rückenmarkstrauma, je nach den neurologischen Ausfällen, die langfristige Prognose bestimmen.

Für den Tierarzt besteht die Herausforderung, initiale Notfallmaßnahmen, eine möglichst frühzeitige Behandlung des SHT, eine fundierte Besitzerkommunikation und die weitere Planung und Durchführung diagnostischer und therapeutischer Maßnahmen koordinieren zu müssen. Hierbei kann die Frage, wie viel Diagnostik zum Ausschluss weiterer Verletzungen notwendig oder sinnvoll ist, nicht allgemeingültig beantwortet werden. Bei einer Katze bieten sich RöntgenÜbersichtsaufnahmen an, um

- Thoraxverletzungen

- ein nicht kardiogenes Lungenödem (Cushing-Reflex)

Konsequenzen eines hohen oder niedrigen arteriellen Kohlendioxidpartialdrucks $\left(\mathrm{P}_{\mathrm{a}} \mathrm{CO}_{2}\right)$ für das Gehirn

$\uparrow \mathrm{paCO}_{2} \rightarrow$ zerebrale Vasodilatation $\rightarrow \uparrow$ zerebrales Blutvolumen $\rightarrow \uparrow$ intrakranieller Druck

$\downarrow \mathrm{paCO}_{2} \rightarrow$ zerebrale Vasokonstriktion $\rightarrow \downarrow$ zerebraler Blutfluss $\rightarrow$ zerebrale $\mathrm{O}_{2}$-Minderversorgung

Die Hyperventilation auch eines gesunden Patienten kann über diesen Mechanismus zu einer zerebralen Hypoxie führen $\rightarrow$ CAVE: Beatmung ohne adäquate Überwachung!

- ein mögliches abdominelles Trauma

- Gliedmaßenfrakturen/-luxationen

- ein offensichtliches Wirbelsäulentrauma auszuschließen bzw. zu diagnostizieren. Diese werden durch Laboruntersuchungen ergänzt (Aussagekraft abhängig vom zeitlichen Abstand nach Trauma). Ein gewisses Restrisiko, Verletzungen nicht erfasst zu haben, bleibt jedoch bestehen. Je nach Ausmaß von Begleitverletzungen und neurologischer Schädigung durch das SHT sollte beim Besitzergespräch auch die Euthanasie des Tieres thematisiert werden. Folgende Symptome sind bei einem SHT als prognostisch ungünstig zu beurteilen [13]:

- komatöser oder sich zusehends verschlechternder Bewusstseinszustand

- Enthirnungsstarre (komatös, Opisthotonus, Extensorenspasmus aller Gliedmaßen)

- unkoordiniertes Atemmuster (Cheyne-Stokes-Atmung)

- massive, offene Schädelfrakturen

- stecknadelkopfgroße Pupillen oder beidseitige, nicht licht-responsive Mydriasis

- Status epilepticus

Für eine Objektivierung der neurologischen Beurteilung schlägt Flegel [12] ein an die Humanmedizin (Glasgow Coma Scale) angelehntes Schema vor (Leipziger Coma Scale, > Tab. 2).

\section{"Nur" Kopfverletzung oder SHT - wie unterscheidet sich die Therapie?}

Primäre Therapieziele beim Traumapatienten sind:

- kardiozirkulatorische Stabilisierung

- respiratorische Stabilisierung
- adäquate Analgesie

Diese decken sich weitgehend mit dem schon angesprochenen Prinzip der Vermeidung von Hypoxie, Hyperkapnie, Hypo-/Hypertension beim SHT. Allerdings steht bei dieser Patientengruppe darüber hinausgehend die Verhinderung und Minimierung von Sekundärschäden im Vordergrund der Behandlung. Dadurch ergeben sich trotz vieler Gemeinsamkeiten an einigen Stellen Besonderheiten und Interessenskonflikte.

\section{Kardiozirkulatorische Stabilisierung}

Anders als beim Hund ist für die Katze eine Trias aus Bradykardie, Hypotension und Hypothermie typisch für ein Schockgeschehen. Eine niedrige Herzfrequenz sollte den Tierarzt bei einer traumatisierten Katze deshalb nicht in Sicherheit wiegen, sondern eher warnen. Darüber hinaus kann eine solche Bradykardie auf den Cushing-Reflex zurückgehen und Anzeichen für einen erhöhten intrakraniellen Druck sein.

Hat eine kranke Katze eine Herzfrequenz unter 160 Schlägen/Minute, sollte man an ein Schockgeschehen denken und dies prüfen [17].

\section{Infusionstherapie}

Basis der kardiozirkulatorischen Stabilisierung ist eine adäquate, zielgerichtete Infusionstherapie ( $\vee$ s. Kasten 2 ), die beim SHT-Patienten einen höheren Blutdruck anstrebt als bei den übrigen Traumapatienten. Leider ist die Überwachung des Blutdrucks beim Kleintier problembehaftet, so deuten neuere Untersuchun- 
Tab. 2 Leipziger Coma Scale. Die maximale Punktzahl für einen gesunden Patienten beträgt 18 Punkte. Werden weniger als 9 Punkte erreicht, ist die Prognose des Tieres ungünstig (Flegel, [12]).

\begin{tabular}{|l|l|}
\hline Bewusstsein & Punkte \\
\hline normal & 5 \\
\hline unangemessene Reaktion & 4 \\
\hline schläft, lässt sich aber durch Geräusche wecken & 3 \\
\hline schläft, lässt sich aber durch Schmerz wecken & 2 \\
\hline keine Reaktion (Koma) & 1 \\
\hline Motorik & \\
\hline normaler Gang & 5 \\
\hline ohne Hilfe gehfähig & 4 \\
\hline nicht gehfähige Tetraparese/Hemiparese & 3 \\
\hline intermittierender Opisthotonus, Extensorenspasmus & 2 \\
\hline permanenter Opisthotonus, Extensorenspasmus & 1 \\
\hline Pupillengröße & 2 \\
\hline beide Pupillen mittlere Größe & 5 \\
\hline bilateral mittelgradige Miosis & 4 \\
\hline Anisokorie & 3 \\
\hline beide Pupillen stecknadelkopfgroß & 2 \\
\hline bilaterale Mydriasis (kein Pupillenlichtreflex) & 1 \\
\hline Summe & \\
\hline
\end{tabular}

gen darauf hin, dass auch das lange Zeit als verlässlich geltende UltraschallDoppler-Verfahren nicht ausreichend genau arbeitet $[6,28,32]$.

Mittel der 1. Wahl ist die balancierte Vollelektrolytlösung (keine glukosehaltige Lösung verwenden!). In der 1 . Stunde können bis zu 45-60 ml/kg KM verabreicht werden. Um eine Volumenüberladung zu vermeiden, wird für die Katze empfohlen, jeweils 1/4 der Menge (10-15 $\mathrm{ml} / \mathrm{kg} \mathrm{KM}$ ) pro 15 Minuten zu verabreichen und dann den Zustand des Tieres neu zu evaluieren [17].

Von Nachteil bei den kristalloiden Lösun-

Therapieziele der Infusionstherapie bei der Traumakatze

(Holowaychuk, [17])

- Verbesserung des Allgemeinbefindens

- Herzfrequenz: (85)-160-200/min

- kräftiger peripherer Puls

- (blass-)rosa Schleimhäute

- kapilläre Rückfüllzeit<2 s

- MAP: 60-70 mmHg Bei SHT: 90-120 mmHg (zentraler Venendruck 5-10 $\mathrm{cmH}_{2} \mathrm{O}$ ) hypotone Lösung. Man führt praktisch reines Wasser zu und verstärkt so die Ödembildung. Auch glukosehaltige Elektrolytlösungen sollten vermieden werden, da in hypoxischen Hirnarealen die Glukose anaerob verstoffwechselt wird. Dies führt zu einer intrazellulären Laktatazidose und verstärkt den sekundären Hirnschaden in vorgeschädigten Arealen. Klinische Studien beim Menschen bestätigen diese Hypothese [23], neuere experimentelle Studien sehen hingegen keinen negativen Einfluss von Glukose [25].

Wegen der Flüssigkeitsverschiebung in den Extravasalraum bei der Gabe von kristalloiden Infusionslösungen sollte man bei Patienten mit SHT die Gabe kolloidaler Infusionslösungen (HES) oder hochkonzentrierter NaCl-Lösung erwägen.

Als kolloidale Infusionslösungen werden heute i.d.R. HydroxyethylstärkePräparate (HES) genutzt. Von diesen werden bei einem Schock-/Traumapatienten 2,5-5 ml/kg KM über 10-15 min verabreicht, die Maximaldosis beträgt 20-40 $\mathrm{ml} / \mathrm{kg} /$ Tag. Bei zu schneller Gabe ist bei der Katze Vomitus beschrieben [17].

HES ist in der Humanmedizin in die Diskussion geraten, nachdem Belege für seine Überlegenheit gegenüber den kristalloiden Lösungen bisher fehlen und Risiken - zumindest bei einigen Patientengruppen - statistisch gesichert wurden. Auch ist die Bedeutung der Speicherung der HES-Moleküle in verschiedenen Geweben nicht geklärt [1, 8, 31]. Aussagekräftige Daten für die Veterinärmedizin zu diesen Aspekten fehlen [1, 8]. Bei Störung der vaskulären Barriere kommt es bei HES ebenfalls zur Flüssigkeitsverschiebung in den Extravasalraum. Anders als bei den kristalloiden Lösungen handelt es sich dabei um einen proteinhaltigen Typ-2-Shift (bis 60\%) [31].

Beim hämorrhagischen Schock führt hochkonzentrierte Kochsalzlösung (7,5\%) zur schnellen Restitution der zentralen und peripheren Hämodynamik [22]. Erreicht wird dies durch einen Flüssigkeitsstrom von interstitiell und intrazellulär nach intravasal. Damit erfolgt der Flüssigkeitsstrom bei dieser Infusionslösung im Vergleich zu der bisher angesprochenen in die entgegengesetzte Richtung. 
Hochkonzentrierte Kochsalzlösung ist eine wirkungsvolle Alternative zu Mannitol zur Senkung des Hirndrucks und diesem möglicherweise sogar überlegen $[5,20,35]$.

Hochkonzentrierte Kochsalzlösung hat einen positiven Effekt auf die Hämodynamik und den intrakraniellen Druck bei Katzen mit SHT.

Verabreicht werden bei der Katze 2-4 ml/kg KM über 5-10(-20) min [15]. Nach sehr schneller Injektion wird eine Bronchokonstriktion beschrieben [17]. Hochkonzentrierte Kochsalzlösung führt zwar zu einer raschen Stabilisierung der Hämodynamik, aufgrund der relativ kurzen Effektdauer (20 min) muss sich jedoch eine bedarfsgerechte Volumentherapie mit kristalloiden oder kolloidalen Infusionslösungen anschließen.

Bei einem sehr hohen Blutverlust ist unter Umständen eine Bluttransfusion nötig (bei der Katze unbedingt Blutgruppe beachten). Steht Blut nicht zur Verfügung, kann die Gabe einer Hämoglobinlösung eine Alternative sein.

\section{Wärmetherapie}

Eine Hypothermie reduziert die Reaktion auf die Katecholamin-vermittelte Sympathikusstimulation und fördert die Entstehung einer Bradykardie. Es entsteht ein Teufelskreis aus Hypothermie, Bradykardie, niedrigem Herzzeitvolumen und weiterem Abfall der Körpertemperatur. Bei einer unterkühlten Katze greift die Infusionstherapie deshalb nicht, es droht sogar eine relative Volumenüberladung. Aus diesen Gründen ist eine frühe Wärmetherapie bei der Katze sehr wichtig $(\rightarrow$ Warmluftdecke, Wärmflasche, Anwärmen der Infusionslösung).

Beim SHT ist jedoch möglicherweise eine Hypothermie vorteilhaft. Sie senkt den Sauerstoffbedarf und damit die Gefahr einer Hypoxie. Der zerebrale Metabolismus ist reduziert und infolgedessen sinken der zerebrale Blutfluss und damit das zerebrale Blutvolumen und das intrakranielle Volumen. Als Konsequenz fällt der intrakranielle Druck. Dieser theoretische Vorteil einer Hypothermie beim SHT konnte am humanen Patienten jedoch nicht bewiesen werden, es resultierte sogar eine höhere Komplikationsrate aus einer therapeutischen Hypothermie [9]. Außerdem ist das adäquate Management eines hypothermen Patienten aufwändig und in der Tiermedizin meist nicht zu leisten, sodass auch beim SHT-Patienten zum Vorteil der kardiozirkulatorischen Funktion eine Wärmetherapie erfolgen sollte.

\section{.konkret}

Eine Hyperthermie ist beim Patienten mit SHT unbedingt zu vermeiden.

\section{Sauerstoff}

Wie wichtig im Hinblick auf die kardiovaskuläre Situation eine ausreichende Sauerstoffaufnahme ist, macht die moderne Definition des Schocks klar. Schock ist definiert als akutes Missverhältnis zwischen Sauerstoffangebot und Sauerstoffbedarf im Gewebe infolge Verminderung der nutritiven Durchblutung lebenswichtiger Organe mit der Konsequenz funktioneller und struktureller hypoxischer Gewebsveränderungen. Dieses Missverhältnis gilt es zu beseitigen.

Das Sauerstoffangebot in Gewebe hängt vom Herzzeitvolumen und dem arteriellen Sauerstoffgehalt ab ( s. Kasten 3 ). Es gibt somit zwei Stellglieder, die genutzt werden können, um die Situation in der Peripherie zu bessern:

- Wiederherstellung/Besserung des Herzzeitvolumens durch eine Infusionstherapie

- Erhöhung des Sauerstoffgehalts im arteriellen Blut

Bietet man dem Traumapatienten eine erhöhte inspiratorische Sauerstoffkonzentration und damit einen erhöhten Sauerstoffpartialdruck an, sichert dies eine normale arterielle Sauerstoffsättigung, also die optimale Beladung des Hämoglobins mit Sauerstoff (im Normalfall auch bei Raumluftatmung gegeben). Darüber hinaus wird die Menge des physikalisch gelösten Sauerstoffs erhöht. Zwar beträgt diese maximal $2 \mathrm{ml} / 100 \mathrm{ml}$ Blut, diese Menge entspricht jedoch etwa der Hälfte des Sauerstoffbedarfs in der Peripherie. Auf diese Weise kann ein niedriges Herzzeitvolumen, aber auch ein reduzierter Hämoglobingehalt (maximal ein Hämoglobin-Abfall um ca. 1,5 g/dl oder ca. $1 \mathrm{mmol} / \mathrm{l}$ Blut) teilweise kompensiert werden. Allerdings wird der maximale Effekt nur bei einer inspiratorischen Sauerstoffkonzentration von 100 Vol.-\% und einer ungestörten Sauerstoffdiffusion in der Lunge erreicht.

Ein Patient mit einer schlechten Kreislauffunktion profitiert von einer Sauerstoffgabe. Er kann den physikalisch gelösten Sauerstoff als „add-on“ nutzen.

\section{Respiratorische Stabilisierung}

Die Gabe von Sauerstoff ist natürlich auch eine Maßnahme zur respiratorischen Stabilisierung, so gleicht eine Erhöhung der inspiratorischen Sauerstoffkonzentration auf 30 Vol.-\% ein auf 50\% reduziertes Atemminutenvolumen aus [27]. Unbedingt beachtet werden muss, dass die bei einer Hypoventilation gleichzeitig bestehende Störung der Kohlendioxidabgabe damit nicht beeinflusst wird. Sie kann nur über eine Normalisierung des Atemzeitvolumens $(\rightarrow$ Beatmung) erreicht werden.

Besteht beim Traumapatienten durch Flüssigkeitseinlagerung in der Lunge (Blutung in die Alveole, Lungenkontusion, Lungenödem) eine Diffusionsstörung, bewirkt eine Erhöhung der Sauerstoffkonzentration in der Einatemluft ein größeres Partialdruckgefälle zwischen Alveole und Blut und damit eine bessere Diffusion des Sauerstoffs in das arterielle Blut. Auch in diesem Fall profitiert der Patient.

Viele Patienten mit Störungen der Ventilation profitieren von einer Gabe von Sauerstoff. Die Abgabe von Kohlendioxid kann jedoch nur durch die Normalisierung des Atemzeitvolumens beeinflusst werden.

Die suffiziente Verabreichung von Sauerstoff ist beim nicht intubierten Patien-

Sauerstoffangebot im Gewebe $=$ Herzzeitvolumen $\times$ arterieller $\mathrm{O}_{2}$-Gehalt

Arterieller $\mathrm{O}_{2}$-Gehalt $=$ chemisch gebundener $\mathrm{O}_{2}+$ physikalisch gelöster $\mathrm{O}_{2}$

$$
=\mathrm{Hb} \times 1,39 \times \mathrm{saO}_{2}+0,003 \times \mathrm{paO}_{2}
$$


ten jedoch ein Problem. Mit Nasalkathetern $(\rightarrow$ partielle Verlegung der Atemwege, bei kleinen Tieren nicht unproblematisch, vor allem bei Einengung der Nase durch Trauma/Schwellung), Trachealkatheter ( $\rightarrow$ Reiz bei hohem Gasfluss, Hustenreiz, Platzierung nur in Narkose oder am bewusstlosen Tier möglich), Sauerstoffmasken ( $\rightarrow$ Stress), Kopfkammern (mit Frischhaltefolie verschlossener Halskragen $\rightarrow$ Stress) und Sauerstoffboxen (CAVE: Wärmeentwicklung) ist es i.d.R. schwierig, konstant Konzentrationen über 30 Vol.- \% zu erreichen.

Weitere Maßnahmen zur Stabilisierung des Gaswechsels sind abhängig von den vorliegenden (Begleit-)Traumata und der Schwere der Atemstörung, sie reichen bis zur Intubation und Beatmung. Bei Gesichtsschädelverletzungen kann es zu einer Verlegung der Atemwege kommen, aus diesem Grund muss die Notwendigkeit einer Intubation sorgfältig geprüft werden. Allerdings erfordert diese eine Narkose (es sei denn, der Patient ist schon bewusstlos) und macht in dieser Indikation nur Sinn, wenn in adäquater Zeit eine Besserung der lokalen Situation erreicht werden kann. Vielen Tieren mit Verletzungen im Bereich der Nase kann man Erleichterung verschaffen, indem man Blutkrusten und eingetrocknetes Sekret von den Nares entfernt und/oder die Nase vorsichtig absaugt. Handelsübliches Nasenspray (Oxymetazolin, Xylometazolin u.ä.) kann versucht werden, um ein Abschwellen der Schleimhaut in der Nase zu erreichen (CAVE: Dosis, Stress).

Bei einer Katze mit SHT sollte man aufgrund der Zusammenhänge in $\mathbf{A b b} . \mathbf{4}$ eine Hyperkapnie vermeiden. Eine starke Hypokapnie sollte ebenfalls wegen der Gefahr der Gewebshypoxie (bedingt durch die zerebrale Vasokonstriktion und die Verschiebung der Sauerstoffbindungskurve nach links $\rightarrow$ Sauerstoff wird im Gewebe schlechter abgegeben) und einer schlechteren Prognose vermieden werden.

Angestrebt wird eine Normo- oder moderate Hyperventilation $\left(\mathrm{p}_{\mathrm{a}} \mathrm{CO}_{2}: 30-35\right.$ $\mathrm{mmHg}$ ) $[24,26]$. Anders als in der Humanmedizin fehlt dem Tierarzt in vielen Fällen die Möglichkeit zu kontrollieren, ob diese Zielwerte erreicht werden. Eine arterielle Blutgasanalyse oder alternativ Kapnografie am intubierten Tier ist dafür notwendig. Außerdem stellt sich für den Tierarzt die Frage, ob er bei einem nicht bewusstlosen Tier gegebenenfalls eine Narkose einleitet, um es intubieren und ventilieren zu können. Die Risiken und die notwendigen Ressourcen (vor allem im Hinblick auf eine möglicherweise länger dauernde Ventilation) müssen kritisch überdacht werden.

\section{Adäquate Schmerztherapie Opioide}

Bei Traumapatienten sind die Opioide aufgrund der guten Schmerzausschaltung und der relativ geringen HerzKreislauf-Wirkungen Mittel der 1. Wahl. Da es in der Zwischenzeit eine Reihe zugelassener Präparate für die Katze gibt ( Tab.3), ist eine adäquate und individuell angepasste Schmerztherapie möglich.

Opioide gelten als „kreislaufschonend“, sie können jedoch eine Bradykardie induzieren. Bei einem kreislaufinstabilen Patienten kann dies problematisch sein, zur Therapie wird ein Parasympatholytikum eingesetzt. Opioide können bei der Katze eine Hyperthermie verursachen. Eine hohe Körpertemperatur sollte beim SHT jedoch unbedingt vermieden werden.
Opioide, die an $\mu$-Rezeptoren agonistisch wirken (Methadon, Fentanyl, Buprenorphin), beeinflussen die Regulation der Atmung. Infolgedessen reagiert der Körper auf einen Anstieg des arteriellen Kohlendioxidpartialdrucks nicht mit einem adäquaten Anstieg des Atemminutenvolumens und der arterielle Kohlendioxidpartialdruck steigt. Genau dies sollte beim SHT jedoch verhindert werden ( $\vee$ Abb.4). Das am $\mu$-Rezeptor antagonistisch wirkende Butorphanol ist deutlich weniger atemdepressiv und kann deswegen beim Patienten mit Schädel-Hirn- oder Thoraxtrauma als alternatives Analgetikum erwogen werden. Allerdings ist seine schmerzausschaltende Wirkung ebenfalls deutlich geringer. Hat der Patient starke Schmerzen, steht der Tierarzt also vor einem Entscheidungsdilemma.

\section{NSAIDs}

Klassische nicht steroidale Analgetika dürfen nur bei kreislaufstabilen Patienten eingesetzt werden $(\rightarrow$ Hemmung der Prostaglandinsynthese $\rightarrow$ Beeinflussung der Regulation der Nierendurchblutung $\rightarrow$ evtl. Entstehung einer Niereninsuffizienz).

Metamizol (20-30 mg/kg KM i.v.) gilt als sehr potent und hinsichtlich der Nierendurchblutung als unkritisch. Allerdings muss unbedingt auf eine sehr langsame Injektion (über $3 \mathrm{~min} \rightarrow$ Injektionslösung verdünnen) geachtet werden, um einen Blutdruckabfall zu vermeiden.

\section{Ketamin}

Auch beim SHT besteht für niedrige Dosen von Ketamin keine Kontraindikation (Ladedosis: 0,1-0,5 mg/mg KM, Dauertropfinfusion: $0,1-1 \mathrm{mg} / \mathrm{kg} / \mathrm{h}$ i.v.). Auf Anzeichen einer Atemdepression sollte geachtet werden.

Tab. 3 Opioide, die bei der Katze angewendet werden können.

\begin{tabular}{|lllll}
\hline Opioid & Opioid-Klasse & Indikation & Wirkdauer & Dosis \\
\hline Methadon & $\mu$-Rezeptor-Agonist & starke Schmerzen & $4 \mathrm{~h}$ & $0,1-0,3(-0,5) \mathrm{mg} / \mathrm{kg} \mathrm{s.c.,} \mathrm{i.m.}$ \\
\hline Fentanyl* & $\mu$-Rezeptor-Agonist & starke Schmerzen & $20-30 \mathrm{~min}$ & $\begin{array}{l}1-2 \mu \mathrm{g} / \mathrm{kg} \mathrm{i.v.} \\
1-7 \mu \mathrm{g} / \mathrm{kg} / \mathrm{h} \text { i.v. }\end{array}$ \\
\hline Buprenorphin & partieller $\mu$-Rezeptor-Agonist & $\begin{array}{l}\text { schwache bis mittel- } \\
\text { starke Schmerzen }\end{array}$ & $4-12 \mathrm{~h}$ & $\begin{array}{l}10-20 \mu \mathrm{g} / \mathrm{kg} \mathrm{s.c.,} \mathrm{i.m.,} \mathrm{i.v.} \\
(\text { Eintritt der Wirkung nach } 30-45 \mathrm{~min})\end{array}$ \\
\hline Butorphanol & Antagonist/Agonist & schwache Schmerzen & bis $4 \mathrm{~h}$ & $0,1-0,4(-0,8) \mathrm{mg} / \mathrm{kg} \mathrm{KM} \mathrm{s.c.,} \mathrm{i.m.,} \mathrm{i.v.}$ \\
\hline
\end{tabular}

* Zulassung in Deutschland nur für den Hund. 


\section{Lidocain}

Beim Menschen gilt die kontinuierliche Verabreichung von Lidocain als vorteilhaft beim SHT. Untersuchungen zu dieser Fragestellung gibt es für die Katze nicht. Jedoch wird in der Anästhesie die intravenöse Verabreichung von Lidocain bei der Katze (Ladedosis: 0,25-0,75 mg/kg KM i.v., Erhaltung bis $2 \mathrm{mg} / \mathrm{kg} / \mathrm{h}$ i.v.) wegen der negativen Herz-KreislaufWirkungen eher als kritisch angesehen [29].

Therapieziel beim SHT ist die Verhinderung und Minimierung von Sekundärschäden. Therapiemaßnahmen sind die Aufrechterhaltung eines physiologischen Blutdrucks, die Sicherung der Oxygenierung und die Reduktion des intrakraniellen Druckes.

\section{Senkung des intrakraniellen Druckes}

Eine einfache Maßnahme zur Drucksenkung ist eine leichte Kopfhochlagerung (30 Grad). Sie führt zu einer Steigerung des venösen Abflusses aus dem Gehirn und damit zu einer Verminderung des zerebralen Blutvolumens. Im Idealfall resultiert eine Abnahme des intrakraniellen Volumens und daraus folgend auch des Druckes. Daten zur Effizienz dieser Maßnahme in der Veterinärmedizin fehlen, sie wird jedoch auch für die Katze empfohlen [11, 15]. Zusätzlich sollte darauf geachtet werden, dass der Hals der Katze nicht abknickt oder die Vena jugularis komprimiert wird (Halskragen, Halsband), da dies den venösen Abfluss behindert.

Eine weitere einfach durchführbare Maßnahme ist eine Osmotherapie mit hochkonzentrierter Kochsalzlösung (s. o.) oder Mannitol (0,5-2 g/kg i.v. über 10-30 min) [11, 15]. Mannitol führt zu einer passageren unspezifischen Dehydratation des Gewebes und senkt die Liquorproduktion. Es verbessert außerdem die rheologischen Eigenschaften des Blutes, dies verbessert die Mikroperfusion im Hirngewebe und damit dessen Sauerstoffversorgung. Durch die Zunahme des intravasalen Volumens steigt die Auswurfleistung des Herzens, was wiederum einen positiven Einfluss auf die zerebrale Durchblutung hat. Empfohlen wird eine Bolusgabe, keine kontinuier- liche Infusion. Eine zu schnelle Infusion oder große Boli können eine Vasodilatation und damit einen hinsichtlich der Hirndurchblutung unerwünschten Blutdruckabfall verursachen. Für den osmotischen Effekt des Mannitols ist eine intakte Blut-Hirn-Schranke Voraussetzung. Ist diese defekt, kann eine Zunahme des Ödems resultieren (ReboundEffekt) [34].

\section{konkret}

Mannitol gilt heute beim Vorliegen einer intrakraniellen Blutung nicht mehr als kontraindiziert.

Außerdem kann die Abhängigkeit des Durchmessers der zerebralen Gefäße vom arteriellen Kohlendioxidpartialdruck ( $\triangleright$ Abb.4) zur Senkung des intrakraniellen Druckes genutzt werden. Der Patient wird dazu normo- oder moderat hyperventiliert $\left(\mathrm{p}_{\mathrm{a}} \mathrm{CO}_{2} \quad 30-35 \mathrm{mmHg}\right)$ [24,26]. Eine starke Hyperventilation birgt durch die entstehende zerebrale Vasokonstriktion die Gefahr einer zerebralen Hypoxie, sie bleibt jedoch als Ultima Ratio, wenn der intrakranielle Druck mit keiner anderen Maßnahme zu senken ist. Leider ist der therapeutische Ansatz Beatmung mit relativ viel Aufwand und auch Risiken verbunden: Der Patient muss anästhesiert, intubiert, beatmet und adäquat überwacht sowie gepflegt und möglicherweise ernährt werden. Die Notwendigkeit zur Intubation beim Schädeltrauma wurde in einer neueren Untersuchung beim Hund als negativer prognostischer Faktor ermittelt [33]. Scheinen Lagerung, Osmotherapie und Beatmung keinen ausreichenden Effekt zu erzielen (Beurteilung häufig schwer) bzw. verschlechtert sich der Patient weiter, wäre der nächste therapeutische Schritt das „Barbituratkoma“. Barbiturate senken den zerebralen Stoffwechsel und damit die Hirndurchblutung und das zerebrale Blutvolumen und so im Endeffekt den intrakraniellen Druck ( Abb. 2, A Abb.3). Sie haben außerdem eine antikonvulsive Wirkung. Beschrieben werden zudem eine Hemmung lysozymaler Enzyme und eine Reduktion der Freisetzung freier Sauerstoffradikale. Beides ist hinsichtlich einer zerebralen
Gewebsschädigung potenziell positiv, die klinische Bedeutung ist jedoch nicht hinreichend geklärt. Von Nachteil sind die Kreislaufwirkung der Barbiturate (unerwünschter Blutdruckabfall) und ihr atemdepressiver Effekt $(\rightarrow$ evtl. Beatmung nötig), von Vorteil (Gehirn) bzw. Nachteil (Kreislauf) ist ihre hypotherme Wirkung. Eine solche Maßnahme erfordert eine aufwändige intensivmedizinische Betreuung, auch fehlt in der Tiermedizin (und Humanmedizin) der Beweis ihrer Nützlichkeit $[2,5,11,30]$. Sie sollte deswegen sehr kritisch auch im Hinblick auf die Prognose eines solchen Patienten diskutiert werden. Zwar wird das Barbituratkoma in veterinärmedizinischen Textbüchern regelmäßig erwähnt, es fehlen jedoch Dosierungskonzepte. Beim Menschen werden Thiopental und Methohexital eingesetzt, eine Alternative wäre Pentobarbital (CAVE: lange Wirkung).

Beim Menschen und zunehmend in der Tiermedizin werden operative Verfahren (Dekompressionstrepanation, Kraniotomie) zur Senkung des erhöhten intrakraniellen Druckes eingesetzt, wenn die konservative Therapie ihre Wirkungen verfehlt bzw. die neurologischen Defizite sehr schwerwiegend sind. Diese bleiben jedoch dem Spezialisten vorbehalten.

Treten Krampfanfälle auf, müssen diese unbedingt behandelt werden, um weitere negative Effekte auf das Hirngewebe zu vermeiden. Genutzt werden übliche Therapieschemata mit Diazepam, Phenobarbital, Levetiracetam und Propofol [14].

\section{.konkret}

Glukokortikoide sind beim SHT nicht indiziert!

In humanmedizinischen Untersuchungen zeigte sich ein fehlender Effekt von Glukokortikoiden auf den Schädel-HirnDruck, jedoch ein Anstieg der Letalität [3]. Dieser Beurteilung schließen sich auch veterinärmedizinische Autoren an $[11,15]$.

Sollte eine Anästhesie notwendig sein, variiert das Vorgehen stark abhängig vom Zustand des Tieres, dem Vorliegen 
eines SHT und der notwendigen Operation/Maßnahme. Die Grundprinzipien sind die bei Traumapatienten üblichen, oben angesprochenen, wie die Notwendigkeit einer Stabilisierung und einer adäquaten perioperativen Analgesie, die Vermeidung kreislaufdepressiver Medikamente und die Sicherung des pulmonalen Gaswechsels.

Auch wenn sie hinsichtlich der Prognose relativ positiv ist, ist eine Feststellung von Hippokrates (zitiert nach [13]) als Fazit gut geeignet: Keine Kopfverletzung ist so schwerwiegend, dass sie hoffnungslos ist, noch ist sie so banal, dass man sie ignorieren darf.

\section{Online zu finden unter}

http://dx.doi.org/10.1055/s-0041-108152

\section{Literatur}

1 Adamik KN, Yozova ID, Rgenscheit N. Controversies in the use of hydroxyethyl starch solutions in small animal emergency and critical care. J Vet Emerg Crit Care 2015; 25: $20-47$

2 Alali A. Process of Care and Outcome of Critically III Patients with Traumatic Brain Injury [PhD-These]. Toronto: University of Toronto; 2014. Im Internet: https://tspace. library.utoronto.ca/bitstream/1807/68347/1/ Alali_Abdulaziz_201406_PhD_thesis.pdf; Stand: 26.06.2015

3 Alderson P, Roberts I. Corticosteroids for acute traumatic brain Injury. Cochrane Database Syst Rev 2005. Im Internet: http:/| www.bibliotecacochrane.com/PDF/ CD000196.pdf; Stand: 26.06.2015

4 Alef M. Zur Nah-Infrarot-Spektroskopie bei Hund und Katze: experimentelle und klinische Untersuchungen zur perioperativen Überwachung sowie zu den Auswirkungen der Anästhesie auf den zerebralen Sauerstoffstatus [Habilitation]. Leipzig: veterinärmedizinische Fakultät der Universität Leipzig; 2002. Im Internet: http://www.qucosa.de/ fileadmin/data/qucosa/documents/3449/ nirs_alef2002_habil.pdf; Stand: 26.06.2015

5 Battison C, Andrews PJ, Graham C, Petty T. Randomized, controlled trial on the effect of a $20 \%$ mannitol solution and a $7.5 \%$ saline $/ 6 \%$ dextran solution on increased intracranial pressure after brain injury. Crit Care Med 2005; 33: 196-202, 257-258

6 Bosiack AP, Mann FA, Dodam JR et al. Comparison of ultrasonic Doppler flow monitor, oscillometric, and direct arterial blood pressure measurements in ill dogs. J Vet Emerg Crit Care (San Antonio) 2010; 20: 207-215

7 Brandt L. Tapia-Syndrom. Seltene Komplikation der Atemwegssicherung. Anästhesist 2015; 64: 122-127
8 Cazzolli D, Prittie J. The crystalloid-colloid debate: consequences of resuscitation fluid selection in veterinary critical care. J Vet Emerg Crit Care 2015; 25: 6-19

9 Clifton GL, Valadka A, Zygun D et al. Very early hypothermia induction in patients with severe brain injury (the National Acute Brain Injury Study: Hypothermia II): a randomised trial. Lancet Neurol 2011; 10: 131-139

10 Deutsche Gesellschaft für Neurochirurgie. Leitlinie Schädel-Hirn-Trauma im Erwachsenenalter. Im Internet: http://www.awmf.org/ uploads/tx_szleitlinien/008-001_S2e_ Schaedel-Hirn-Trauma_im_Erwachsenenalter_leitlinientext_abgelaufen.pdf; Stand: 28.05.2015

11 Dewey CW, Fletcher DJ. Head Trauma Management. In: Dewey CW. A Practical Guide to Canine \& Feline Neurology. $2^{\text {nd }}$ ed. Ames, lowa, USA: Wiley-Blackwell; 2008: 221-236

12 Flegel T. Schädel-Hirn-Trauma: der tierische Blickwinkel. In: Rackwitz R, Pees M, Aschenbach JR, Gäbel G, Hrsg. Leipziger Blaue Hefte. 7. Leipziger Tierärztekongress Tagungsband 3. Berlin: Lehmanns Media; 2014: 545-549

13 Flegel T, Preuß M. SHT beim Kleintier - Da brummt der Schädel. HundKatzePferd 2014; 6: 22-25

14 Forterre F, Jurina K. Erkrankungen des Nervensystems. In: Lutz H, Kohn B, Forterre F, Hrsg. Krankheiten der Katze. Stuttgart: Enke; 2015: 803-845

15 Freeman C, Platt S. Head Trauma. In: Platt S, Garosi L, eds. Small Animal Neurological Emergencies. London: Manson Publishing; 2012: 363-382

16 Herzer G, Trimmel H. Neuroanästhesie Grundlagen der perioperativen Betreuung. Anaesthesist 2010; 59: 371-384

17 Holowaychuk MK. Management of Shock in Cats: They're Not Just Small Dogs. Im Internet: http://c.ymcdn.com/sites/www. invma.org/resource/resmgr/2015_speaker_ proccedings_/dr._marie_k._holowaychuk_ man.pdf; Stand: 28.05.2015

18 Hudetz JA, Pagel PS. Neuroprotection by ketamine: a review of the experimental and clinical evidence. J Cardiothorac Vasc Anesth 2010; 24: 131-142

19 Jurati TA, Stephan SE, Stepha AE, Sobottka SB. Akutversorgung des Patienten mit schwerem Schädel-Hirn-Trauma. Anästhesist 2015; 64: 159-174

20 Kempski O, Obert C, Mainka T et al. "Small volume resuscitation" as treatment of cerebral blood flow disturbances and increased ICP in trauma and ischemia. Acta Neurochir Suppl 1996; 66: 114-117

21 Kolata RJ, Kraut NH, Johnston DE. Patterns of trauma in urban dogs and cats: a study of 1000 cases. J Am Vet Med Assoc 1974; 164: 499-502

22 Kreimeier U, Christ F, Frey L et al. Smallvolume resuscitation beim hypovolämischen Schock. Konzeption, experimentelle und klinische Ergebnisse - eine Standortbestimmung. Anaesthesist 1997; 46: 309-328
23 Liu-DeRyke X, Collingridge DS, Orme J et al. Clinical impact of early hyperglycemia during acute phase of traumatic brain injury. Neurocrit Care 2009; 11: 151-157

24 Ma XD, Willumsen L, Hauerberg J et al. Effects of Graded Hyperventilation on Cerebral Blood Flow Autoregulation in Experimental Subarachnoid Hemorrhage. J Cereb Blood Flow Metab 2000; 20: 718-725

25 Moro N, Ghavium S, Harris NG et al. Glucose administration after traumatic brain injury improves cerebral metabolism and reduces secondary neuronal injury. Brain Research 2013; 1535: 124-136

26 Muizelaar JP, Marmarou A, Ward JD et al. Adverse effects of prolonged hyperventilation in patients with severe head injury: a randomized clinical trial. J Neurosurg 1991; 75: 731-739

27 Nunn JF. Applied Respiratory Physiology. London: Butterworth; 1997

28 Petric AD, Petra Z, Jerneja S, Alenka S. Comparison of high definition oscillometric and Doppler ultrasonic devices for measuring blood pressure in anaesthetised cats. J Feline Med Surg 2010; 12: 731-737

29 Pypendop BH, Ilkiw JE. Assessment of the hemodynamic effects of lidocaine administered IV in isoflurane-anesthetized cats. Am J Vet Res 2005; 66: 661-668

30 Roberts I, Sydenham E. Barbiturates for acute traumatic brain injury. Cochrane Database Syst Rev 2012; doi: 10.1002/14651858. CD000033.pub2; Stand: 29.06.2015

31 Roessler M, Bode K, Bauer M. Volumentherapie bei Hämorrhagie. Anästhesist 2014; 63: 730-744

32 Seliškar A, Zrimšek P, Sredenšek J, Petrič AD. Comparison of high definition oscillometric and Doppler ultrasound devices with invasive blood pressure in anaesthetized dogs. Vet Anaesth Analg 2013; 40: 21-27

33 Sharma D, Holowaychuck MK. Retrospective evaluation of prognostic indicators in dogs with head trauma: 72 cases (January-March 2011). J Vet Emerg Crit Care 2015; doi: 10.1111/ vec. 12328

34 Shawkat H, Westwood MM, Mortimer A. Mannitol: a review of its clinical uses. Contin Educ Anaesth Crit Care Pain 2012; 12: 82-85

35 Wakai A, McCabe A, Roberts I, Schierhout G. Mannitol for acute traumatic brain injury. Cochrane Database Syst Rev 2013; DOI: 10.1002/14651858.CD001049.pub5

Prof. Dr. med. vet Michaele Alef

Klinik für Kleintiere

Veterinärmedizinische Fakultät Universität

Leipzig

An den Tierkliniken 23

04103 Leipzig 


\section{Fragebogen}

\section{Frage 1}

Die Durchblutung des Gehirns ist über einen weiten Blutdruckbereich unabhängig von diesem. Welcher Blutdruckwert stellt in etwa die untere Grenze dieses Bereich dar?

a. ein systolischer Blutdruck von $50 \mathrm{mmHg}$

b. ein systolischer Blutdruck von $150 \mathrm{mmHg}$

c. ein mittlerer arterieller Blutdruck von $150 \mathrm{mmHg}$

d. ein mittlerer arterieller Blutdruck von $50 \mathrm{mmHg}$

e. ein mittlerer arterieller Blutdruck von $100 \mathrm{mmHg}$

\section{Frage 2}

Wie ist der Zusammenhang von zerebralem Perfusionsdruck, Blutdruck und intrakraniellem Druck?

a. zerebraler Perfusionsdruck= mittlerer arterieller Blutdruck - intrakranieller Druck

b. zerebraler Perfusionsdruck = intrakranieller Druck - mittlerer arterieller Blutdruck

c. zerebraler Perfusionsdruck= systolischer Blutdruck - intrakranieller Druck

d. zerebraler Perfusionsdruck = intrakranieller Druck - diastolischer Blutdruck

e. zerebraler Perfusionsdruck=intrakranieller Druck - zentralvenöser Blutdruck

\section{Frage 3}

Ihnen wird eine Katze nach einem Sturz aus dem 5. Stock vorgestellt. Der Patient ist nicht ansprechbar, zeigt eine Tachykardie, einen schwachen Puls, blasse Schleimhäute, eine Hypothermie, eine beidseitige Miosis und einen Opisthotonus. Sie diagnostizieren ein Schockgeschehen und vermuten ein SHT. Welche Infusionslösung scheint in dieser Situation nahezu ideal zu sein?

a. bilanzierte Vollelektrolytlösung

b. Hydroxyethylstärke-Lösung

c. Mannitol

d. $5 \%$ ige Glukoselösung

e. hochkonzentrierte Kochsalzlösung

\section{Frage 4}

Wie hoch ist die Reduktion des Atemminutenvolumens, die Sie mit einer inspiratorischen Sauerstoffkonzentration von $30 \mathrm{Vol}$. \% in etwa kompensieren können?
a. $10 \%$
b. $20 \%$
c. $3 \%$
d. $50 \%$
e. $70 \%$

\section{Frage 5}

Welches Symptom tritt beim CushingReflex immer auf?

a. Bluthochdruck

b. Bradykardie

c. Arrhythmien

d. Lungenödem

e. Atemstörung

\section{Frage 6}

Opioide gelten auch beim SchädelHirn-Trauma als Analgetika der Wahl. Beachtet werden müssen jedoch die von ihnen ausgelöste Atemdepression und die eventuell auftretende Bradykardie. Welche weitere mögliche Opioid-Nebenwirkung muss speziell bei der Katze berücksichtigt werden? a. Hypotension

b. Hyperoxie

c. Hyperthermie

d. Hypertonie

e. Hypokapnie

\section{Frage 7}

Bei der Katze ist eine Trias aus Bradykardie, Hypotension und Hypothermie typisch für ein Schockgeschehen. Ab welcher Herzfrequenz sollte man bei einer kranken Katze schon an ein Schockgeschehen denken?
a. 80 Schläge/min
b. 100 Schläge/min
c. 120 Schläge/min
d. 140 Schläge/min
e. 160 Schläge/min

\section{Frage 8}

Welche Gruppe von Analgetika ist bei einem Traumapatienten mit instabilen Kreislaufverhältnissen kontraindiziert?

a. Opioide

b. nicht steroidale Antiphlogistika

c. NMDA-Rezeptor-Antagonisten

d. nicht saure Nicht-Opioid-Analgetika

e. Lokalanästhetika

\section{Frage 9}

Bei einem Patienten mit SHT sollte man einen hohen arteriellen Kohlendioxidpartialdruck unbedingt vermeiden. Was ist der Hauptgrund dafür?
a. zerebrale Vasodilatation
b. respiratorische Depression
c. zerebrale Hypoxie
d. respiratorische Azidose
e. zerebrale Vasokonstriktion

\section{Frage 10}

Welche Lagerungsmaßnahme wird beim Vorliegen eines SHT zur Reduktion des intrakraniellen Druckes empfohlen?
a. $45^{\circ}$ Oberkörper-Hochlagerung
b. $30^{\circ}$ Oberkörper-Hochlagerung
c. Oberkörper-Neutrallagerung
d. $45^{\circ}$ Oberkörper-Tieflagerung
e. $30^{\circ}$ Oberkörper-Tieflagerung 


\section{Kopftrauma bei der Katze}

Michaele Alef

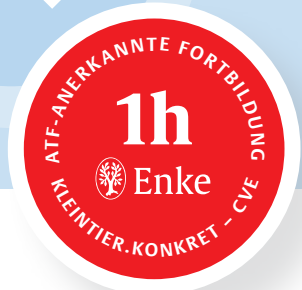

A Lernerfolgskontrolle

\section{Bitte kreuzen Sie die richtigen Antworten an! Es ist jeweils nur 1 Antwort pro Frage richtig!}

\section{B Teilnehmer}

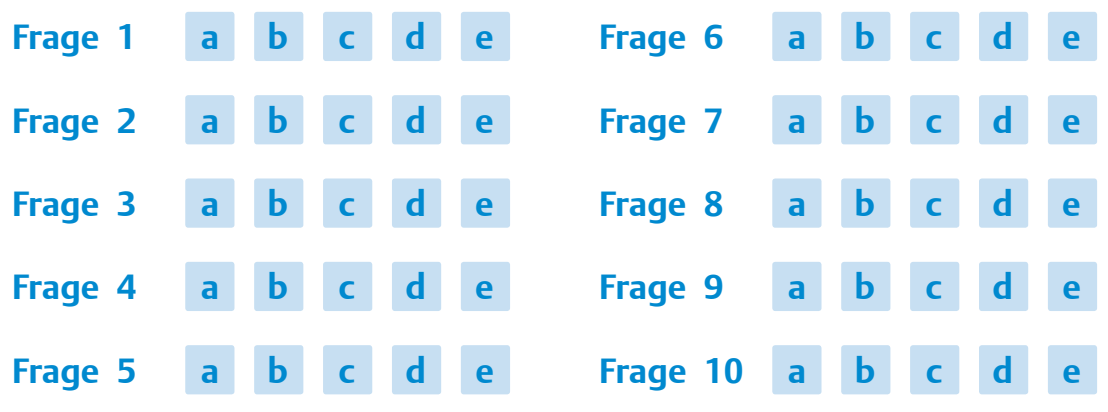

\section{Ihr Ergebnis wird vom Verlag ausgefüllt}

\section{E Erklärung}

Ich versichere, dass ich die Beantwortung der

Fragen selbst und ohne Hilfe durchgeführt habe.

Sie haben von $\_\square$ bestanden und 1 ATF-Stunde erhalten.

Stuttgart, den

D Teilnahmebedingungen für Abonnenten der kleintier konkret kostenlos

Für diese Fortbildungseinheit können Sie 1 ATFFortbildungsstunde anerkannt bekommen. Hierfür

- müssen mindestens 70 \% der Fragen richtig beantwortet sein.

- muss der Antwortbogen vollständig ausgefüllt sein. Unvollständig ausgefüllte Bögen können nicht berücksichtigt werden!

\section{- muss im markierten Feld* Ihre Abonnen- tennummer eingetragen oder eine kleintier konkret-CVE-Wertmarke aufgeklebt sein.}

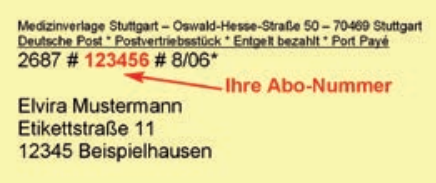

kleintier konkret-CVE-Wertmarken für NichtAbonnenten können beim Verlag zu folgenden Bedingungen erworben werden: 6erPackWertmarken, Preis 49,95€ inkl. MWSt., Artikel-Nr. 903000.

Bitte richten Sie die Bestellungen an: MVS Medizinverlage Stuttgart,

KundenServiceCenter Buch, Postfach 3011 20, 70451 Stuttgart.

* Nicht-Abonnenten bitte hier kleintier konkret-CVE-Wertmarke aufkleben, Abonnenten bitte Abonnentennummer eintragen 